\title{
The Patient Admission Scheduling of an Ophthalmic Hospital Using Genetic Algorithm
}

\author{
Liming Zhang, Huiyou Chang, Ruitian Xu \\ Department of Computer Science, Sun Yat-sen University \\ Guangzhou, China \\ Corresponding Author: Huiyou Chang \\ 13922413531@139.com
}

\begin{abstract}
Currently, FCFS scheduling method is widely used in hospitals for patient admission scheduling, which ignores the impacts of patient length of stay and surgery arrangement on the usage of hospital resources. This paper proposes a more comprehensive mathematical model and evaluation mechanism for the patient admission scheduling of an ophthalmic hospital. A genetic algorithm (GA) is proposed to optimize the model, which can provide detailed scheduling of patient admission in the hospital for everyday. The result is compared with that of the traditional FCFS method, which indicates that the GA helps to reduce the preoperative waiting time for patients. Besides, GA can provide different kinds of scheduling for the hospital to select by adjusting the relative weights of different objectives in the algorithm.
\end{abstract}

Keywords- Ophthalmic hospital, patient admission scheduling, genetic algorithm

\section{INTRODUCTION (HEADING 1)}

Due to the deficiency of medical equipments and medical personnel, hospitals are always overcrowded, coming with a long line. An effective and efficient patient admission scheduling has a positive influence on hospitals such as resource availability, management efficiency, cost effectiveness, and society influence, to name a few. FCFS (First come, First serve) basis is widely used for nonemergency cases in hospitals these days, which is a service policy whereby patients are able to get the admission to hospitals in the order they arrive. This policy only considers the minimum average waiting time for hospital admission, whereas fails to take into account the differences among patients' in-hospital time, and the bad impact on the availability of hospital resource brought by the variation of operation arrangements. Thereby it is unfavorable for effectiveness of the hospital management and can't shorten waiting time for hospital admission.

This paper establishes a comparatively perfect decisionmaking model of the patient admission scheduling and bases on overall analysis on waiting times for hospital admission, waiting times for surgery, the length of waiting list for hospital admission and vacancy rate of hospital beds etc, and it also introduces Genetic Algorithm to optimize patient admission scheduling. Considering the rationality of FCFS basis for hospital admission with the same type of disease, when applying Genetic Algorithm to solve the problem of patient hospital admission scheduling, we will focus on diseases selection rather than patient selection. That means, we only calculate the number of the patients with the same type of disease in a day, and apply the FCFS basis for patients of the same type, which ensure that we are able to arrange patient admissions scientifically and decrease the complexity of solution of this problem solved by Genetic Algorithm dramatically.Genetic Algorithm (GA) is a random heuristic search algorithm based on the evolutionary ideas of natural selection and genetics [1][2], its characteristics present as follow: (1) Its principal is clear and the iterative process is not only simple but also understandable, which evolves through the three operators: selection, crossover, mutation; (2) It is widely used since it operates directly on the solution to a given problem, which is not required the mathematical characteristics such as continuity and differentiability; (3) It uses self-organization to search the problem space and self-adaption to adjust the search direction, which is analogous that there is no extra rule or regulation instituted to direct the search in a black box. (4) It processes preferable global optimum and robustness. With these characteristics, Genetic algorithm has been arisen widespread attention recently, and applied in different field [3]-[6] successfully. At the same time, it can also be applied in the intelligent patient admission scheduling. In this paper, we conducted our experiments in the Ophthalmic Hospital in Beijing, scheduling the admission for the patients with cataract disease (one eye, both eyes), with retina disease, with glaucoma disease and with ocular trauma, respectively. We compared the performance of the model proposes in this paper with the performance of FCFS basis used to be adopted by the hospital, and the experimental results show that our model is more effective in shortening the waiting times for surgery. The rest of this paper is organized as follows. Chapter 2 introduces the model of the patient admission scheduling. Chapter 3 describes the primary Genetic Algorithm. Chapter 4 presents how to apply the Genetic Algorithm to optimize patient admission scheduling. Chapter 5 compares the experimental test results. At last, Chapter 6 concludes this paper.

\section{THE MODEL OF PATIENT ADMISSION SCHEDULING}

Admission scheduling of the waiting lists requires considering the arrangement for surgery date and in-hospital time (in-hospital time usually is affected by the day on which 
a patient will have surgery). Taking a certain ophthalmic hospital as an example, the surgeries in this hospital are primarily classified into four categories, cataract surgery, retina surgery, glaucoma surgery and ocular trauma surgery. The process of the surgery is as follow: (1) Patients with cataract disease can separate into two categories, one is the patients who needs surgery only for one eye, the other is those who need surgery for both eyes, both of whose surgeries are comparatively simple, so it only takes one or two days for preoperative evaluation and preparation. All the cataract surgeries are arranged on Mondays and Wednesdays, if some patients have cataracts in both eyes, they will have the surgery on one eye at a time. One surgery is on Monday while the other is on Wednesday. (2) Other surgeries (nonemergency), which are relatively complexity, take two to three day the preoperative evaluation and preparation after admission and a long period of observation after surgery. Besides, considering the shift schedule of surgeons, the other surgeries (non-emergency) are not arranged on the same day with cataract surgeries. (3) Patients with ocular trauma are usually emergency cases, who do not need admission scheduling, as they would be admitted as long as there are beds available in the hospital. On the next day of their admission, the patients will have the surgery, which will not conflict with other surgeries.

In order to establish the model of patient admission schedule of this ophthalmic hospital, we have to assume some reasonable hypothesis on problems, so as to exclude limitation and unnecessary interfere, which may lead to an effective establishment of the model and an easy solving process and extension. According to the data of all kinds of patients from July $13^{\text {th }}, 2008$ to September $11^{\text {th }}, 2008$, and considering the facility and resource of the hospital, we assume the model as follow:

1) Patients with same type of disease will be admitted according to the order of their arrivals.

2) Patients with same type of disease will have surgery according to the order of their admissions.

3) Cataract surgery will be arranged only on Mondays and Wednesdays.

4) The other surgery (non-emergency) will be arranged on any day except Monday and Wednesday.

5) Patients with ocular trauma will be transferred to other hospitals (instead of staying in the waiting list) if no beds available.

6) Patients with ocular trauma will be admitted into the hospital the next day of their admission if there is a bed available.

7) Sufficiency of surgical and medical resource allows us to ignore the constraints of surgery conditions while scheduling ocular surgeries.

\section{A. Symbol Definition and Decision Various}

The symbols used to describe the model are defined as Table 1 presented. On the next day after considering the availability of hospital resource, a part of patients on the waiting list will be admitted. The emergency patients do not need admission scheduling, as they will be admitted as long as there are beds available. The number of the emergency patients, who will admitted on the next day, can be assured, which is defined as $N_{0}^{\prime}=\min \left\{N_{0}, M-M^{\prime}+O\right\}$. After the emergency patients admitted, the number of the left available beds is $M \mathrm{e}_{1}=M-M^{\prime}+O-N_{0}{ }^{\prime}$, which are available for the patient with cataract disease, with retina disease, with glaucoma disease, and with ocular trauma, respectively. Assume that there are $n$ patients will be admitted on the next day, and the number of available beds on the next day is $M \mathrm{e}_{2}=M \mathrm{e}_{1}-n$

Table 1 Symbol Definition

\begin{tabular}{|c|c|}
\hline Symbol & Definition \\
\hline$M$ & The total number of the beds in the hospital \\
\hline$M^{\prime}$ & The number of the occupied beds the first day \\
\hline $\begin{array}{c}P_{1} \backslash P_{2} \backslash P_{3} \backslash \\
P_{4}\end{array}$ & $\begin{array}{l}\text { The number of patients with monocular cataract } \backslash \\
\text { binocular cataract \glaucoma|retinopathy }\end{array}$ \\
\hline$O$ & $\begin{array}{l}\text { The estimated number of the patients who discharge the } \\
\text { next day }\end{array}$ \\
\hline$N_{0}$ & $\begin{array}{l}\text { The number of the emergency patients who arrives the } \\
\text { first day }\end{array}$ \\
\hline$N_{0}^{\prime}$ & $\begin{array}{l}\text { The number of the emergency patients admitted the } \\
\text { next day, } N_{0}^{\prime}=\min \left\{N_{0}, M-M^{\prime}+O\right\}\end{array}$ \\
\hline$N$ & $\begin{array}{l}\text { The total number of the patients on the admission } \\
\text { waiting list (non- emergency cases), } \\
N=N_{1}+N_{2}+N_{3}+N_{4}\end{array}$ \\
\hline $\begin{array}{c}N_{1} \backslash N_{2} \backslash N_{3} \backslash \\
N_{4}\end{array}$ & 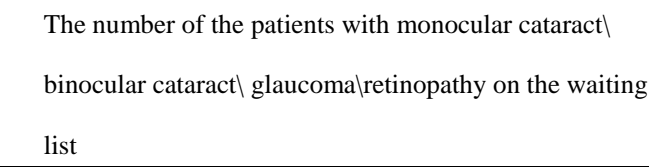 \\
\hline$n$ & $\begin{array}{l}\text { The total number of the patients admitted the next day } \\
\text { (non-emergency cases), } n=n_{1}+n_{2}+n_{3}+n_{4}\end{array}$ \\
\hline $\begin{array}{c}n_{1} \backslash n_{2} \backslash n_{3} \backslash \\
n_{4}\end{array}$ & 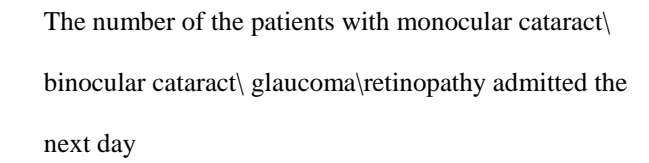 \\
\hline$M \mathrm{e}_{1}$ & $\begin{array}{l}\text { The estimated number of bed available for the patients } \\
\text { before they admitted the next day, }\end{array}$ \\
\hline
\end{tabular}




\begin{tabular}{c|c}
\hline & $M \mathrm{e}_{1}=M-M^{\prime}+O-N_{0}{ }^{\prime}$ \\
\hline $\mathrm{e}_{2}$ & $\begin{array}{c}\text { The estimated number of bed available for the patients } \\
\text { after they admitted the next day, } M \mathrm{e}_{2}=M \mathrm{e}_{1}-n\end{array}$ \\
\hline$T_{1}$ & $\begin{array}{c}\text { The total length of the waiting time for the left patient in } \\
\text { the waiting list }\end{array}$ \\
\hline$T_{2}$ & $\begin{array}{l}\text { The number of days of the new arrival patients waiting } \\
\text { for surgeries }\end{array}$ \\
\hline$W$ & $\begin{array}{l}\text { The period of the next day (if } W=0 \text { denotes Sunday, } \\
\text { then } 1 \text { denotes Monday and so on) }\end{array}$ \\
\hline$b_{i}$ & $\begin{array}{l}\text { Whether or not the } i^{\text {th }} \text { patient in the waiting list admitted } \\
(1 \text { denotes No, } 0 \text { denotes Yes) }\end{array}$ \\
\hline$c_{i}$ & $\begin{array}{l}\text { The number of days before the } i^{\text {th }} \text { patient in the waiting } \\
\text { list admitted }\end{array}$ \\
\hline
\end{tabular}

The decision various of the model are $n_{1}, n_{2}, n_{3}$ and $n_{4}$, denote the number of the patients with monocular cataract $\backslash$ binocular cataract $\backslash$ glaucoma\retinopathy admitted the next day.

\section{B. Evaluation System}

- The average waiting time for patient admissions

Because of the randomness of patient arrival and the constraint of resource and equipments in hospital, it is inevitable that patients have to wait for their admissions. The average waiting time for the admission directly affects the satisfaction and the choice of patients, the society influence of a hospital and economic benefit. Thus the hospital should decrease the average waiting time for patient admission as possible.

In the model, waiting time for admission can be calculated by the equation as follow,

$$
Y_{1}=T_{1} /(N-n)
$$

Where $T_{1}=\sum_{i=1}^{N} c_{i} \times b_{i}$ is the total waiting time for admissions for the patients on the waiting list.

- The Preoperative Average Waiting Time of Admitted Patients

It is a significant factor of evaluation system that preoperative waiting time for surgery as admitted patients. Because of the routine check-up as the time of admission and constraints of surgical resource, it is evitable that patient have to wait for their surgeries for a period of time, which is affected by patient admission scheduling. A simple instant is not far to seek. If a patient has cataracts in both eyes, admitted in the weekend, he might only wait for two days before he has surgeries. If the patient is admitted on Monday, he might wait for a whole week for his surgeries. The waiting time will decrease patient satisfaction, while it is a waste of beds and other resource of the hospital. As a result, preoperative waiting time will be minimized by means of reasonable admission scheduling.

In the model, the average preoperative waiting time for new patients can calculate by the follow formula:

$$
Y_{2}=T_{2} / n
$$

Where T2 is an estimated total waiting time for the new patients. According to model, cataract surgeries only arrange on Mondays and Wednesdays, while other surgeries arranges on any day except Mondays and Wednesday, so the methods calculate different preoperative waiting time is various. Here is the value of $\mathrm{T} 2$ :

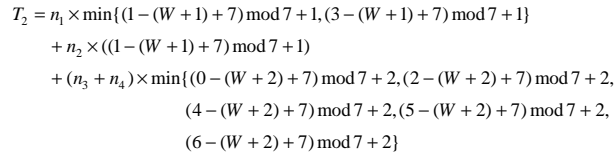

Where is composed by three different terms, the first term is the estimated preoperative waiting time for monocular cataract surgery, the second term is the estimated preoperative waiting time for binocular cataract surgery, and the third term is the estimated preoperative waiting time for glaucoma and retinopathy surgery. $W+1$ denotes that it takes at least one day for patients with cataract disease for their preoperative preparations, $W+2$ denotes that it takes at least two days for patients with glaucoma and retinopathy disease for their preoperative preparations.

- Number of patients on the waiting list

Too many patients on the waiting list could influence the public order in hospital and prolong the average waiting time for admission, which also becomes a problem in that ophthalmic hospital nowadays. So when designing a new scheduling model, we need to consider how to shorten the length of waiting list for patient admission, which is:

$$
Y_{3}=N-n
$$

- Vacancy Rate of Bed

A high vacancy rate of hospital bed means a low occupied rate of hospital bed, which is an extremely waste for hospital. As the definitions shows in Table $1, \mathrm{M}$ is the total number of the hospital beds, and $\mathrm{Me} 2$ is the estimated number of the left beds after scheduling the new admitted patient the next day, thus the vacancy rate of the beds can be calculated by following formula:

$$
Y_{4}=M \mathrm{e}_{2} / M
$$

- $\quad$ The fairness to Different Patients

Although the complexity of different kinds of surgeries and the in-hospital time are various, as a medical institution, a hospital should take the social responsibility and maintain the fairness to different patients admission scheduling while pursuing effectiveness and benefits. It also affects public satisfaction to hospitals. After one admission scheduling, the number of the patients with cataract disease is 
$\mathrm{P} 1+\mathrm{P} 2+\mathrm{n} 1+\mathrm{n} 2$, the number of the patients with glaucoma disease is $\mathrm{P} 3+\mathrm{n} 3$ and the number of the patients with retinopathy disease is $\mathrm{P} 4+\mathrm{n} 4$. The fairness to different patients' admission scheduling can be measured by the standard deviation of these three numbers or other methods.

- $\quad$ Profit of Hospital

The profit of hospital evolves many aspects such as government policies, hospital effectiveness, to name a few, which requires taking a great of factors into account, but this is not the pivot of this paper, so we are not going to quantitative analysis here.

\section{Comprehensive Evaluation Value}

In the evaluation system of this paper, the first four standards are the major ones for model evaluation. Because of the interaction among these standards, any of these standards is insufficient to evaluate the model reasonably and effectively, thus we need to establish a comprehensive evaluation value for this evaluation system. This comprehensive evaluation value is composed by the average waiting time for patient admission, the preoperative waiting time, the number of patients on the waiting list, the vacancy rate of the beds. $Z$ is the comprehensive evaluation value, which can denote as:

$$
Z=w_{1} f_{1}+w_{2} f_{2}+w_{3} f_{3}+w_{4} f_{4}=\sum_{i=1}^{4} w_{i} f_{i}
$$

Where $f_{1}, f_{2}, f_{3}, f_{4}$ can be computed by normalizing $Y_{1}$, $Y_{2}, Y_{3}, Y_{4}$, and adopt the arc cotangent transformation, the formula presented as follow:

$$
f_{i}=\arctan \left(Y_{i}\right) \times 2 / \pi, \quad i=1,2,3,4
$$

Where the coefficient $w_{1}, w_{2}, w_{3}, w_{4}$ are defined for the four objectives functions $f_{1}, f_{2}, f_{3}, f_{4}$, respectively. The weights are able to adjust based on the different situation, which reflects the flexibility of the evaluation system.

From the formula of the comprehensive evaluation value, we can conclude based on this evaluation system that the smaller the comprehensive evaluation value is, the more reasonable the evaluation system.

\section{GENETIC AlgORITHM AND ITS DESCRIPTION}

Genetic Algorithm (GA) is a computing method that simulates the natural evolution. It uses "Population" to express the set of possible solutions. "Individual" (Chromosome) denotes a possible solution to the specific problem. "Fitness Value" is assigned to each solution representing the ability of an individual to "complete". The iterative process evolves three operators, which are selection operator, crossover operator and mutation operator, and applies the genetic operations on the population, which could keep evolving until the best individual good enough to satisfy the requirement of the problem. The flow chat of the Genetic Algorithm is presented as Fig.3.

\section{A. Selection Operation}

Selection Operation gives preference to better individuals and allow them to pass on their genes to the next generation in proportion to their fitness, which is realized by roulette technology. The individuals with high fitness have high probability to pass on their genes to the next generation, while the least fit individuals have a tendency to die out. Selection operation is favorable for increasing the average value of population the motive of the evolution. But selection operation of all individuals tends to be similar among the population, which means it will decrease the diversity of the population. The diversity of the group is maintained by crossover operation and especially by mutation operation.

\section{B. Crossover Operation}

Each chromosome goes through the crossover process with a certain probability randomly from the population, of which gene fragments exchange after matching. Crossover has analogy to genetic recombination, since the offspring may inherit good characteristics from both parent chromosomes and process better fitness value. Crossover operation generates new chromosomes for genetic evolution. Notice that it is a process of gene pass and inherit rather than of gene creation.

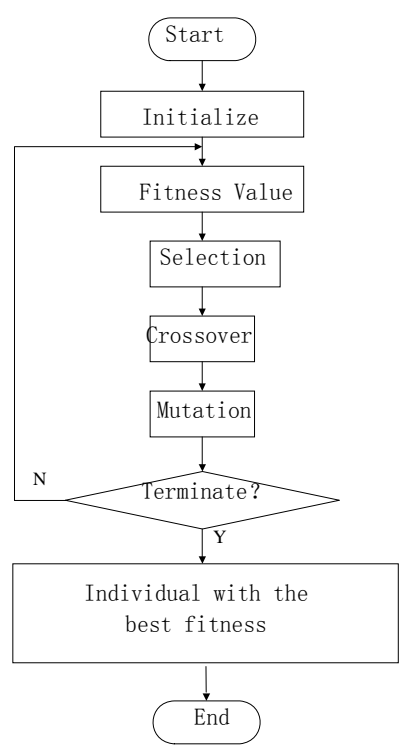

Fig 1 Flow Chat of Genetic Algorithm

\section{Mutation Operation}

Mutation operation is a process to create new genes, which randomly decides whether or not the gene in a certain chromosome from the population will be changed based on the mutation probability. With genes that represented in a way of real number, one of the mutation operations randomly adds a new real number within domain to replace 
the original gene. The new gene improves the global search ability and inhibits premature convergence of local optimum.

\section{The Application of THE Genetic AlgORITHM ON PATIENT ADMISSION SCHEDULING}

\section{A. Chromosome Coding}

Considering applying Genetic Algorithm on the problems in the real world, the first thing need to do is to solve the problem of chromosome coding, which is how to encode the variables represented as chromosome (Gene Sequence) to optimize by population evolution. In the patient admission scheduling model describing in Chapter 2, the solution to the model is about the next day admission scheduling of all kinds of patients, $n_{1}$ denotes the number of patients have cataract disease on one eye, $n_{2}$ denotes the number of patients have cataract disease on both eyes, $n_{3}$ denotes the number of patients have glaucoma disease, and $n_{4}$ denotes the number of patients have retina disease, each encoding chromosome presented as follow:

$$
X=\left[n_{1}, n_{2}, n_{3}, n_{4}\right]
$$

Please notice that the number of patients with ocular trauma admitted the next day is not a part of gene sequence, since that depends on the number of outpatients with ocular trauma and the beds available the day before, hence it is fixed instead of a decision variable and unnecessary to optimize.

Each variable in the chromosome has its own domain. Genetic Algorithm's purpose is to value and combine the variables within their domains and select the optimum solution. Thus after encoding the chromosome, we must set the domain for each gene (variable) in the chromosome, respectively. In the problem of patient admission scheduling, it is required that the number of patients with a certain disease admitted the next day are less than the number of patients with this kind disease on the waiting list, which is :

$$
\begin{array}{rlrl}
\quad 0 & \leq n_{1} \leq N_{1} & , & 0 \leq n_{2} \leq N_{2} \\
0 \leq n_{4} \leq N_{4} & \text { (9) }
\end{array}
$$

\section{B. Fitness Function}

A significant prerequisite to apply the Genetic Algorithm for the optimum solution is to construct the fitness function that represents how close a given design solution is to the set of aims. Four standards of patient admission scheduling model are deduced in the Chapter 2 that are the average waiting time for patient admissions $Y_{1}=T_{1} /(N-n)$, the preoperative average waiting time of admitted patients $Y_{2}=T_{2} / n$, number of patients on the waiting list $Y_{3}=N-n$, vacancy rate of bed $Y_{4}=M_{2} / M$, and the definitions and the computing methods of $T_{1}, T_{2}, N, n, M_{2}$ have been introduced in Chapter 2. With a careful analysis, the mathematical meaning of Standard $Y_{3}$ and Standard $Y_{4}$ tends to be the same, which means when $Y_{3}$ is minimize, $Y_{4}$ is also minimized at the same time. Thus, we can leave optimization of $Y_{4}$ out of account. As we discussed above, when Genetic Algorithm is applied in the patient admission scheduling problem, the objective function of evaluating individual fitness can define as the weighted sum of normalizing $Y_{1} 、 Y_{2}$ and $Y_{3}$. The formula presented as follow:

$$
\begin{aligned}
& \begin{array}{l}
Z=w_{1} f_{1}+w_{2} f_{2}+w_{3} f_{3} \\
\text { where } \\
f_{1}=\arctan \left(Y_{1}\right) \times 2 / \pi
\end{array} \\
& f_{2}=\arctan \left(Y_{2}\right) \times 2 / \pi, \quad f_{3}=\arctan \left(Y_{3}\right) \times 2 / \pi, \text { and } w_{1}, \\
& w_{2}, \quad w_{3} \text { are weights. }
\end{aligned}
$$

\section{Constraint Condition}

The problem we proposed in the paper is required to satisfy one constraint condition: the number of admitted patients is less than the number of the next day beds available, which is,

$n_{1}+n_{2}+n_{3}+n_{4} \leq M e_{1}$

The solution satisfied this constraint condition called the feasible solution to the problem. Otherwise, the solution called infeasible solution. It is possible to cause an infeasible solution during the crossover operation and mutation operation to the population, but since this constraint condition is not a linear inequality constraint, we can adjust the strategies [7] that adjust the infeasible solutions to feasible solutions, to ensure the efficiency of the algorithm.

\section{EXPERIMENT RESULT AND COMPARABLE ANALYSIS}

\section{A. Experiment Result}

The experiment data is from a certain ophthalmic hospital in Beijing, where there are 79 beds in the inpatient department. The algorithm is based on the number of occupied beds and the waiting list of admission on the day of September $11^{\text {th }}, 2008$, whose purpose is to optimize a bed scheduling on the day of September $11^{\text {th }}, 2008$ with an estimated number of discharged patients. The program can compute the estimated number of discharged patients on the day of $12^{\text {th }}: O=0,1,2, \ldots, 79 ; 80$ probabilities of patient admission scheduling. Here listed only 15 group of the result data (estimated number of discharge patient is $O=11,12,13, \ldots, 25$ ), as presented in Table 2 .

\section{B. Standard Comparison}

For the very reason that this hospital used to adopt FCFS strategy in the patient admission scheduling, we compared and analyzed the experiment results of GA with those of FCFS utilizing the same data of the admission scheduling. The comparison of the four standards is showed as below. 
From the Table 3 , as the increasing estimated number of discharge patients, we can conclude that the average waiting time for the patient admissions and the length of waiting list decrease. From the comparison between FCFS and the Genetic Algorithm, the average waiting time for the admission by GA is longer than that by FCFS. From the formula $Y_{1}=T_{1} /(N-n)$, the average waiting time of admission is the quotient of the total waiting time among the waiting list $T_{1}$ and the number of patients on the waiting list $N-n$. Under the condition of the same number of admitted patients, because the FCFS is the strategy that admits patients according to the order of their arrivals, the total waiting time must be shorter than those computed by any other method. Thus the average waiting time by FCFS is the shortest. From the average preoperative waiting time, GA performs better than FCFS. Because the average preoperative waiting time is closely interrelated to the type of disease, the date of the admission and the operation arrangement and so on that are not considered in the FCFS strategy, while GA fully takes into account the preoperative waiting time of different type of disease and the arrangement of the surgery during optimizing $Y_{2}=T_{2} / n$. In the light of this standard, the result by GA is much best than the result by FCFS.

Moreover, because the number of the beds in the hospital is fixed and the hospital statistic data shows that the demand of the bed always exceeds its supply in the inpatient department, so the number of patient on the waiting list decreases as the number of discharged patient increases, and the vacancy rate of bed reaches $100 \%$.

\section{The Flexible Analysis of Weight}

Actually, different weight coefficient combinations delegate importance of the different objects. Thus each hospital can adjust its own weight coefficients. GA provides a solution with high flexibility, which can adjust all kinds of different situation and is universal by its model. Table 4 shows the test instance where estimated number of discharged patient $O=15$ and presents the different admission scheduling by different objective weight coefficients.

\section{CONCLUSION}

This paper proposes a mathematical model of patient admission scheduling for the ophthalmic hospital, which fully takes waiting time for admission, preoperative waiting time, and length of waiting list in the in-patient department and the occupancy rate of bed and so on. In the new evaluation system, it optimizes the model by GA and establishes the patient admission scheduling intelligently based on the occupancy rate of bed and the waiting list. Since the admission of patient with same disease is scheduled by FCFS strategy, this paper transfers patient selection to disease selection and decrease the complexity of the problem, leading to a much more efficient solution by GA. The solution shows that GA provides a scheduling with shorter length of waiting list and lower vacancy rate of bed and significantly decrease the preoperative waiting time at the same time, which is favorable for availability of hospital resource and management efficiency. We will improve the model by considering the fairness among the patients with different diseases and the hospital profits in the future.

\section{ACKNOWLEDGMENT}

This project is funded by the National Nature Scientific and Guangdong Fund (2009-2012), U0835002.

\section{REFERENCES}

[1] J. H. Holland, Adaption in Natural and Artificial Systems, University of Michigan Press, Ann Arbor, 1975.

[2] D. E. Goldberg, Genetic Algorithms in Search, Optimization, and Machine Learning, Addison-Wesley, Reading, 1989.

[3] J. Zhang, W. L. Lo, and H. S.-H. Chung, "Pseudo-coevolutionary Genetic Algorithms for Power Electronic Circuits Optimization”, IEEE Transactions on Systems, Man, and Cybernetics--Part C, Vol.36, No.4, pp. 590-598, July 2006.

[4] J. Zhang, H. S.-H. Chung and W. L. Lo, “Clustering-Based Adaptive Crossover and Mutation Probabilities for Genetic Algorithms”, IEEE Transactions on Evolutionary Computation, Vol.11, No.3, pp. 326335, June 2007.

[5] X.-M. Hu, J. Zhang, Y. Yu, Y.-L. Li, X.-N. Luo, and Y.-H. Shi, "Hybrid Genetic Algorithm Using a Forward Encoding Scheme for Lifetime Maximization of Wireless Sensor Networks", IEEE Transactions on Evolutionary Computation, vol. 14, no. 5, pp. 766781, 2010.

[6] S.-H. Hur, R. Katebi, and A. Taylor, "Modeling and Control of a Plastic Film Manufacturing Web Process," IEEE Transactions on Industrial Informatics, vol. 7, no. 2, pp. 171-178, 2011.

[7] P. Chootinan and A. Chen, "Constraint handling in genetic algorithms using a gradient-based repair method," Computers \& operations research, vol. 33, no. 8, pp. 2263-2281, 2006. 
TABLE 2 THE ADMISSION SCHEDULING ON $12^{\text {TH }}$ BASED ON DIFFERENT ESTIMATED NUMBER OF DISCHARGED PATIENTS

\begin{tabular}{|c|c|c|c|c|c|c|}
\hline \multicolumn{7}{|c|}{ The admission scheduling on 12th } \\
\hline \multirow{2}{*}{$\begin{array}{c}\text { estimated } \\
\text { number of } \\
\text { discharged } \\
\text { patients }\end{array}$} & \multicolumn{5}{|c|}{ estimated number of discharged patients } & \multirow[b]{2}{*}{ The new patients’ No. on the waiting list } \\
\hline & Trauma & $\begin{array}{l}\text { Cataract } \\
\text { (one eye) }\end{array}$ & $\begin{array}{c}\text { Cataract } \\
\text { (both } \\
\text { eyes) }\end{array}$ & Retina & Glaucoma & \\
\hline 11 & 1 & 0 & 0 & 6 & 4 & $2,3,4,5,8,10,11,12,13,17,97$ \\
\hline 12 & 1 & 0 & 0 & 7 & 4 & $2,3,4,5,8,10,11,12,13,15,17,97$ \\
\hline 13 & 1 & 0 & 0 & 8 & 4 & $2,3,4,5,8,10,11,12,13,15,16,17,97$ \\
\hline 14 & 1 & 0 & 2 & 8 & 3 & $1,2,3,4,5,6,8,10,11,12,13,15,16,97$ \\
\hline 15 & 1 & 0 & 2 & 9 & 3 & $1,2,3,4,5,6,8,10,11,12,13,15,16,21,97$ \\
\hline 16 & 1 & 0 & 2 & 10 & 3 & $1,2,3,4,5,6,8,10,11,12,13,15,16,21,23,97$ \\
\hline 17 & 1 & 0 & 2 & 10 & 4 & $1,2,3,4,5,6,8,10,11,12,13,15,16,17,21,23,97$ \\
\hline 18 & 1 & 0 & 2 & 11 & 4 & $1,2,3,4,5,6,8,10,11,12,13,15,16,17,21,23,24,97$ \\
\hline 19 & 1 & 1 & 2 & 11 & 4 & $1,2,3,4,5,6,7,8,10,11,12,13,15,16,17,21,23,24,97$ \\
\hline 20 & 1 & 2 & 2 & 11 & 4 & $1,2,3,4,5,6,7,8,10,11,12,13,14,15,16,17,21,23,24,97$ \\
\hline 21 & 1 & 2 & 3 & 11 & 4 & $1,2,3,4,5,6,7,8,9,10,11,12,13,14,15,16,17,21,23,24,97$ \\
\hline 22 & 1 & 2 & 4 & 11 & 4 & $1,2,3,4,5,6,7,8,9,10,11,12,13,14,15,16,17,18,21,23,24,97$ \\
\hline 23 & 1 & 3 & 4 & 11 & 4 & $1,2,3,4,5,6,7,8,9,10,11,12,13,14,15,16,17,18,21,22,23,24,97$ \\
\hline 24 & 1 & 2 & 6 & 11 & 4 & $1,2,3,4,5,6,7,8,9,10,11,12,13,14,15,16,17,18,19,20,21,23,24,97$ \\
\hline 25 & 1 & 3 & 6 & 11 & 4 & $1,2,3,4,5,6,7,8,9,10,11,12,13,14,15,16,17,18,19,20,21,22,23,24,97$ \\
\hline
\end{tabular}

TABLE3 THE COMPARISON BETWEEN FCFS AND GA

\begin{tabular}{|c|c|c|c|c|c|c|}
\hline \multirow[t]{2}{*}{$\begin{array}{c}\text { Estimated } \\
\text { Number Of } \\
\text { Discharged } \\
\text { Patients }\end{array}$} & \multicolumn{2}{|c|}{$\begin{array}{l}\text { The average waiting } \\
\text { time for } \\
\text { patient admissions }\end{array}$} & \multicolumn{2}{|c|}{$\begin{array}{c}\text { The Preoperative } \\
\text { Average Waiting } \\
\text { Time of Admitted } \\
\text { Patients }\end{array}$} & \multirow[t]{2}{*}{$\begin{array}{c}\text { Number Of } \\
\text { Patients On The } \\
\text { Waiting List } \\
\text { FCFS/GA }\end{array}$} & \multirow[t]{2}{*}{$\begin{array}{c}\text { Vacancy Rate of } \\
\text { Beds } \\
\text { FCFS/GA }\end{array}$} \\
\hline & FCFS & GA & FCFS & GA & & \\
\hline 11 & 6.154 & 6.308 & 2.364 & 2.000 & 91 & $100 \%$ \\
\hline 12 & 6.089 & 6.256 & 2.333 & 2.000 & 90 & $100 \%$ \\
\hline 13 & 6.022 & 6.202 & 2.308 & 2.000 & 89 & $100 \%$ \\
\hline 14 & 5.955 & 6.102 & 2.357 & 2.143 & 88 & $100 \%$ \\
\hline 15 & 5.897 & 6.046 & 2.333 & 2.133 & 87 & $100 \%$ \\
\hline 16 & 5.837 & 6.000 & 2.313 & 2.125 & 86 & $100 \%$ \\
\hline 17 & 5.776 & 5.929 & 2.294 & 2.118 & 85 & $100 \%$ \\
\hline 18 & 5.714 & 5.869 & 2.333 & 2.111 & 84 & $100 \%$ \\
\hline 19 & 5.651 & 5.795 & 2.368 & 2.158 & 83 & $100 \%$ \\
\hline 20 & 5.585 & 5.720 & 2.350 & 2.200 & 82 & $100 \%$ \\
\hline 21 & 5.519 & 5.642 & 2.333 & 2.238 & 81 & $100 \%$ \\
\hline 22 & 5.450 & 5.575 & 2.364 & 2.273 & 80 & $100 \%$ \\
\hline 23 & 5.308 & 5.504 & 2.250 & 2.306 & 79 & $100 \%$ \\
\hline 24 & 5.308 & 5.436 & 2.375 & 2.333 & 78 & $100 \%$ \\
\hline 25 & 5.247 & 5.364 & 2.400 & 2.360 & 77 & $100 \%$ \\
\hline
\end{tabular}

TABLE 4 THE FLEXIBLE ANALYSIS BY DIFFERENT WEIGHT COEFFICIENTS WHERE O = 15

\begin{tabular}{|c|c|c|c|c|c||}
\hline \multirow{2}{*}{$\begin{array}{c}\text { weight } \\
\text { coefficient } \\
w_{1}, w_{2}, w_{3}\end{array}$} & \multicolumn{5}{|c||}{ The estimated number of admitted patient } \\
\cline { 2 - 6 } & Trauma & $\begin{array}{c}\text { Cataract (one } \\
\text { eye) }\end{array}$ & $\begin{array}{c}\text { Cataract } \\
\text { (both eyes) }\end{array}$ & Retina & Glaucoma \\
\hline $1,0,0$ & 1 & 2 & 3 & 6 & 3 \\
\hline $0,1,0$ & 1 & 0 & 0 & 7 & 7 \\
\hline $0,0,1$ & 1 & 1 & 6 & 0 & 7 \\
\hline $0.4,0.3,0.3$ & 1 & 0 & 2 & 9 & 3 \\
\hline
\end{tabular}

